\title{
THE DEATH OF AN INDEX THEOREM
}

\author{
MANUEL GONZALES AND ROBIN HARTE
}

(Communicated by Paul S. Muhly)

\begin{abstract}
If the "index theorem" for Fredholm operators sometimes hold in the absence of an index [4], then also it sometimes fails in the presence of and index.
\end{abstract}

0. Call the bounded linear operator $T: X \rightarrow Y$ between normed spaces weakly Fredholm if it is both essentially one-one, in the sense ([3, Definition 6.4.1]) that

$$
T^{-1}(0) \text { is finite dimensional, }
$$

and essentially dense, in the sense ([3, Definition 6.4.1]) that

$$
Y / \operatorname{cl}(T X) \text { is finite dimensional; }
$$

thus $T$ is Fredholm if it is weakly Fredholm and proper, in the sense ([3, Definition 3.2.7]) that

$$
\operatorname{core}(T): X / T^{-1}(0) \rightarrow \operatorname{cl}(T X) \text { is invertible, }
$$

where $\operatorname{core}(T)$ is induced by $T$ in the obvious way. The product of weakly Fredholm operators is Fredholm; we have the implication

$S, T$ essentially one-one $\Rightarrow S T$ essentially one-one $\Rightarrow T$ essentially one-one since $T^{-1}(0) \subseteq(S T)^{-1}(0)$ and

$$
(S T)^{-1}(0) / T^{-1}(0) \cong T(X) \cap S^{-1}(0) \subseteq S^{-1}(0),
$$

and the implication

$$
S, T \text { essentially dense } \Rightarrow S T \text { essentially dense } \Rightarrow S \text { essentially dense }
$$

since

$$
T \text { essentially dense } \Leftrightarrow T^{\dagger} \text { essentially one-one, }
$$

Received by the editors March 10, 1989.

1980 Mathematics Subject Classification (1985 Revision). Primary 47B30. 
where $T^{\dagger}: Y^{\dagger} \rightarrow X^{\dagger}$ is the dual or adjoint of $T: X \rightarrow Y$. If $T: X \rightarrow Y$ is weakly Fredholm then we may define its "index":

$$
\operatorname{index}(T)=\operatorname{dim} T^{-1}(0)-\operatorname{dim} Y / \operatorname{cl}(T X),
$$

where "dim" is the usual linear space dimension. Much if not all of the interest in the index lies in the fact that if $T: X \rightarrow Y$ and $S: Y \rightarrow Z$ are both Fredholm then ([3, Theorem 6.5.4];[5]; [6])

$$
\operatorname{index}(S T)=\operatorname{index}(S)+\operatorname{index}(T) ;
$$

indeed the essence of this extends [4] to pairs $(S, T)$ for which $S, T$ and $S T$ all have "generalized inverses". When (0.9) holds we shall say that the pair $(S, T)$ has the index property. Our first observation is that this does not hold universally:

1. Example. If $T=W: X \rightarrow Y=X$ is one-one and dense and if $S=I-f \odot$ $e: X \rightarrow Z=X$ with

$$
f(e)=1 \text { and } e \notin W(X),
$$

then $(S, T)$ does not have the index property.

Proof. The rank one operator $f \odot e$ sends vectors $x \in X$ into $f(x) e$, and $S=S^{2}$ is a projection, which is Fredholm of index zero. The operator $T$ is "weakly invertible", therefore also has index zero. Since $T$ has dense range there is equality

$$
\operatorname{cl}(S T X)=\operatorname{cl}(S X)
$$

we claim that also

$$
(S T)^{-1}(0)=T^{-1}(0) \text {. }
$$

Indeed if $x \in X$ then $(S T) x=T x-f(T x) e$ and hence, since $e \notin T(X)$,

$$
(S T) x=0 \Rightarrow T x=f(T x) e \Rightarrow f(T x)=0 \Rightarrow T x=0 .
$$

Now $\operatorname{cl}(S X)$ has codimension 1 and $T^{-1}(0)$ has dimension 0 , giving

$$
\operatorname{index}(S T)=0-1 \neq 0+0=\operatorname{index}(S)+\operatorname{index}(T) .
$$

For a specific example take $X=l_{2}$ and $e_{n}=1 / n \quad(n \in \mathbb{N}), f(e)=1$ and then define $(W x)_{n}=e_{n} x_{n} \quad(n \in \mathbb{N})$ for each $x \in X$. Example 1 shows that the index of weakly Fredholm operators is not stable under finite rank perturbation, and not continuous with respect to the operator norm topology:

$$
\operatorname{index}(T-f T \odot e)=\operatorname{index}(S T) \neq \operatorname{index}(T)
$$

and

$$
\text { index }(T-t f T \odot e)=0 \rightarrow 0 \neq \operatorname{index}(S T) \quad \text { as } t \rightarrow 1 \text {. }
$$


The essence of Example 1 extends to more general products:

2. Theorem. If $T: X \rightarrow Y$ is one-one and dense and if $S: Y \rightarrow Z$ is Fredholm of index zero, and satisfies

$$
T(X) \cap S^{-1}(0)=\{0\} \neq S^{-1}(0)
$$

then $(S, T)$ does not have the index property. If in addition $Z=X$ and

$$
S(Y) \subseteq \operatorname{cl}(T S Y)
$$

then $(S T, S T)$ does not have the index property.

Proof. Equality (1.2) holds exactly as in Example 1, and equality (1.3) follows from (2.1) and (0.5). If (2.2) is assumed then also

$$
\operatorname{cl}(S T)^{2}(X)=\operatorname{cl}(S Y) \text { and }(S T)^{-2}(0)=T^{-1}(0)
$$

giving

$$
\operatorname{index}(S T)^{2}=\operatorname{index}(S T) \neq 2 \operatorname{index}(S T) .
$$

For a specific example take the direct sum of each of the operators in Example 1 with the identity $I: \mathbb{C} \rightarrow \mathbb{C}$. The discontinuity of the index is exhibited whenever the condition (2.1) of Theorem 2 is satisfied ([2, Theorem 1.1]), as well as instability with respecit to finite rank perturbation.

We have already observed that if both factors $S$ and $T$ are actually Fredholm then (0.9) holds; the same is clear if either $S$ or $T$ is invertible:

$$
S \text { invertible } \Rightarrow \operatorname{index}(S T)=\operatorname{index}(T)
$$

and

$$
T \text { invertible } \Rightarrow \operatorname{index}(S T)=\operatorname{index}(S) .
$$

We can extend this kind of derivation of $(0.9)$ :

3. Theorem. If $T: X \rightarrow Y$ and $S: Y \rightarrow Z$ are weakly Fredholm and either

$$
T \text { is onto }
$$

or

$$
S \text { is bounded below }
$$

or

$$
S \text { is one-one and } T \text { is dense, }
$$

then $(S, T)$ has the index property.

Proof. If (3.3) holds then the isomorphism

$$
T^{-1}(0) \times S^{-1}(0) \times Z / \operatorname{cl}(S T X) \cong(S T)^{-1}(0) \times Y / \operatorname{cl}(T X) \times Z / \operatorname{cl}(S Y)
$$

is established in three stages: there is equality $(S T)^{-1}(0)=T^{-1}(0)$ since $S$ is one-one, there is equality $Z / \operatorname{cl}(S T X)=Z / \operatorname{cl}(S Y)$ since $T$ is dense, and finally $S^{-1}(0)=\{0\}=Y / \operatorname{cl}(T X)$. Counting dimensions on either side gives 
(0.9). If we assume either that $S$ is one-one or that $T$ is onto then $(0.5)$ holds with equality at the end, giving

$$
(S T)^{-1}(0) / T^{-1}(0) \cong S^{-1}(0)
$$

and hence

$$
\operatorname{dim}(S T)^{-1}(0)=\operatorname{dim} S^{-1}(0)+\operatorname{dim} T^{-1}(0) .
$$

For equality

$$
\operatorname{dim} Z / \operatorname{cl}(S T X)=\operatorname{dim} Z / \operatorname{cl}(S Y)+\operatorname{dim} Y / \operatorname{cl}(T X)
$$

we use the analogue of $(3.5)$ with $\left(T^{\dagger}, S^{\dagger}\right)$ in place of $(S, T)$ : if either $S$ is bounded below so that $S^{\dagger}$ is onto, or $T$ is onto so that $T^{\dagger}$ is one-one, there is equality $S^{\dagger}\left(Z^{\dagger}\right) \cap T^{\dagger-1}(0)=T^{\dagger-1}(0)$. Taking stock, both (3.6) and (3.7) are established separately under each of the conditions (3.1), (3.2) and (3.3).

The second part of Theorem 2 shows that commutativity $S T=T S$ is not sufficient for the index property (0.9):

4. Theorem. If $T: X \rightarrow X$ and $S: X \rightarrow X$ are weakly Fredholm and commute, and if either

$$
T \text { is weakly invertible }
$$

or

$$
S \text { is Fredholm of finite ascent and descent, }
$$

then $(S, T)$ has the index property.

Proof. If $T$ is weakly invertible, in the sense of being one-one and dense, then with no commutativity assumptions there is isomorphism
(4.3) $T^{-1}$
$(0) \times S^{-1}$
$(0) \times X / \mathrm{cl}(S T X) \cong(T S)^{-1}(0)$
$(0) \times X / \operatorname{cl}(T X) \times X / \operatorname{cl}(S X):$

the derivation is very similar to that of (3.4). Specialising to $T S=S T$ now gives (3.4) and hence (0.9). If instead $S$ is Fredholm of finite ascent and descent then ([1, Theorem 3.3]; [3, Theorem 7.3.6]) some power $S^{n}$ has a commuting generalized inverse, and we can write

$$
S^{n}=U P=P U \quad \text { with invertible } U \text { and idempotent } P \text {; }
$$

it now follows that $P$ is in the "double commutant" of $S^{n}$, and in particular commutes with $S, T$ and $S T$. We can therefore write

$$
X=\left(\begin{array}{l}
X_{1} \\
X_{0}
\end{array}\right), \quad P=\left(\begin{array}{cc}
I_{1} & 0 \\
0 & 0
\end{array}\right) \quad \text { and } \quad R=\left(\begin{array}{cc}
R_{1} & 0 \\
0 & R_{0}
\end{array}\right) \quad \text { whenever } R S=S R,
$$

where $X_{1}$ and $X_{0}$ are the range and null space of the projection $P$; in particular $X_{0}$ is finite dimensional, and $S_{1}^{n}$ and hence also $S_{1}$ are invertible. Looking separately at $R(X)$ and $R^{-1}(0)$ shows that

$$
R P=P R \Rightarrow \operatorname{index}(R)=\operatorname{index}\left(R_{1}\right)+\operatorname{index}\left(R_{0}\right):
$$


thus

$$
\left(\begin{array}{c}
\operatorname{index}(T) \\
\operatorname{index}(S) \\
i \operatorname{index}(S T)
\end{array}\right)=\left(\begin{array}{c}
\operatorname{index}\left(T_{1}\right) \\
i \operatorname{index}\left(S_{1}\right) \\
i \operatorname{index}\left(S_{1} T_{1}\right)
\end{array}\right)+\left(\begin{array}{c}
i \operatorname{index}\left(T_{0}\right) \\
i \operatorname{index}\left(S_{0}\right) \\
i \operatorname{dex}\left(S_{0} T_{0}\right)
\end{array}\right) .
$$

Since $X_{0}$ is finite dimensional we must have ([3, Theorem 6.2.6]; [5])

$$
\operatorname{index}\left(T_{0}\right)=\operatorname{index}\left(S_{0}\right)=\operatorname{index}\left(S_{0} T_{0}\right)=0 ;
$$

since $S_{1}$ is invertible (2.5) gives

$$
\operatorname{index}\left(S_{1}\right)=0 \text { and } \operatorname{index}\left(S_{1} T_{1}\right)=\operatorname{index}\left(T_{1}\right) .
$$

It follows that the third entry in each of the columns on the right hand side of (4.7) is the sum of the first two; this must also hold on the left hand side, giving $(0.9)$.

Commutativity cannot be dropped from the assumptions of Theorem 4 , since conditions (4.1) and (4.2) both hold in Example 1; in Theorem 2 the Fredholm operators of index zero are ([2]; [3, Theorem 6.5.2]) just the products of invertible operators and (Fredholm) projections. A variant of the second case of Theorem 4 holds whenever one factor is Fredholm: if $S=S S^{\prime} S$ is Fredholm and $T$ commutes with the projection $P=S^{\prime} S$ then (0.9) holds (use the same decomposition of $X=Y$ as in the proof of Theorem 4), and similarly if instead $T=T T^{\prime} T$ is Fredholm and $S$ commutes with $T T^{\prime}$. We conclude with an example which shows that the commutativity in (4.6) cannot be weakened to the invariance of the range of $P$ under $T$ :

5. Theorem. If $A: X \rightarrow X, B: Y \rightarrow X$ and $D: Y \rightarrow Y$ satisfy $A$ is one-one and dense, and $D$ is dense

and

$$
A(X) \cap B(Y)=\{0\}=B^{-1}(0) \cap D^{-1}(0),
$$

then

$$
\left(\begin{array}{cc}
A & B \\
0 & D
\end{array}\right):\left(\begin{array}{c}
X \\
Y
\end{array}\right) \rightarrow\left(\begin{array}{c}
X \\
Y
\end{array}\right) \text { is one-one and dense. }
$$

Proof. If $x \in X$ and $y \in Y$ there is implication

$$
\begin{aligned}
\left(\begin{array}{ll}
A & B \\
0 & D
\end{array}\right)\left(\begin{array}{l}
x \\
y
\end{array}\right) & =\left(\begin{array}{l}
0 \\
0
\end{array}\right) \Rightarrow A x+B y=0=D y \\
& \Rightarrow A x=B y=0=D y \Rightarrow x=0=y
\end{aligned}
$$

if $f \in X^{\dagger}$ and $g \in Y^{\dagger}$ there is the implication

$$
(f g)\left(\begin{array}{cc}
A & B \\
0 & D
\end{array}\right)=\left(\begin{array}{ll}
0 & 0
\end{array}\right) \Rightarrow f A=0=f B+g D \Rightarrow f=0=g \text {. }
$$

Here $A$ is the restriction of $R=\left(\begin{array}{ll}A & B \\ 0 & D\end{array}\right)$ to the invariant subspace $\left(\begin{array}{l}X \\ 0\end{array}\right) \subseteq\left(\begin{array}{l}X \\ Y\end{array}\right)$, and $D$ the induced quotient mapping; if in particular $A$ and $D$ are both 
weakly Fredholm then so is $R$, and the extended version of (4.6) would be that index $(R)=\operatorname{index}(A)+\operatorname{index}(D)$. Theorem 5 however says that index $(R)=$ 0 when the conditions (5.1) and (5.2) hold; to satisfy them without having index $(D)=0$ we can for example take $X=Y=l_{2}$ and $A=W$ as in Example $1, D=V$ the backward shift (so $V(Y)=Y$ and $\operatorname{dim} V^{-1}(0)=1$ ), and finally $B=f \odot e$ where $e \in X$ is not in the range $W(X)$ and $f \in Y^{\dagger}$ satisfies $f^{-1}(0) \cap V^{-1}(0)=0$.

\section{REFERENCES}

1. R. E. Harte, Fredholm, Weyl and Browder theory, Proc. Roy. Irish Acad. Sect. A 85 (1986), 151-176.

2. __ Regular boundary elements, Proc. Amer. Math. Soc. 99 (1987), 328-330.

3. __ Invertibility and singularity, Dekker, New York, 1988.

4. __ The ghost of an index theorem, Proc. Amer. Math. Soc., 106 (1989), 1031-1034.

5. D. Sarason, The multiplication theorem for Fredholm operators, Amer. Math. Monthly 94 (1987), 68-70.

6. K. W. Yang, Index of Fredholm operators, Proc. Amer. Math. Soc. 41 (1973), 329-330.

Department of Mathematics, Universidad de Cantabria, 39005 Santander, Spain

Department of Mathematics, University College, Cork, Ireland

Current address (Robin Harte): Mathematical Sciences, University of Alaska, Fairbanks, Alaska 99775-1110 